\title{
Consistency Analysis of Intraoperative Blood Loss Assessment Between Surgeons and Anesthesiologists
}

Jian Liu

General Hospital of Ningxia Medical University

HongBao Ma

Ningxia Medical University

Faxuan Wang

Ningxia Medical University

Xuewei Wang

general hospital of ningxia medical university

Chaoran Wang

general hospital of ningxia medical university

Linan Wang

general hospital of ningxia medical university

Yubo Gao

general hospital of ningxia medical university

Ningkui Niu ( $\square$ liujian18372552411@163.com)

Ningxia Medical University

Research article

Keywords: perioperative period, blood loss, consistency

Posted Date: April 27th, 2021

DOl: https://doi.org/10.21203/rs.3.rs-445367/v1

License: (c) (1) This work is licensed under a Creative Commons Attribution 4.0 International License.

Read Full License 


\section{Abstract}

Objective: To explore the consistency between intraoperative blood loss recorded by surgeons, anesthesiologists and standard blood loss.

Methods: The clinical data of 200 patients who underwent posterior lumbar interbody fusion (PLIF) from January 2019 to December 2020 in the Department of Spine Surgery of a Grade III Level A hospital were selected, including 109 males, aged 34-84 years, with an average of $56.40 \pm 11.21$ years, and 91 females, aged 27-78 years, with an average of $57.48 \pm 11.40$ years. The preoperative Hb, HCT, APTT, Pt, INR, intraoperative blood, and fluid infusion of patients were recorded. The standard blood loss of the patients was calculated by the formula and compared with the intraoperative blood loss recorded by the surgeons and the anesthesiologists separately for analysis.

Results: 1 . When the standard blood loss was less than $400 \mathrm{ml}$, there was no statistical difference between the intraoperative blood loss recorded by surgeons and the standard blood loss $(P>0.05)$, which is considered to be consistent. 2. When the standard blood loss was between $400 \mathrm{ml}-800 \mathrm{ml}$, the intraoperative blood loss recorded by surgeons and anesthesiologists was less than the standard blood loss,there was statistical difference between the intraoperative blood loss and the standard blood loss ( $P$ $<0.05$ ), but the intraoperative blood loss recorded by surgeons was more accurate than that recorded by anesthesiologists; 3 . When the standard blood loss was more than $800 \mathrm{ml}$, the intraoperative blood loss recorded by surgeons and anesthesiologists was less than the standard blood loss, There was no consistency between the intraoperative blood loss recorded by anesthesiologists and the standard blood loss $(P<0.05)$, but the intraoperative blood loss recorded by anesthesiologists was more accurate than that recorded by surgeons.

Conclusion: 1. There are differences between surgeons and anesthesiologists in recording intraoperative blood loss. 2. The accurate recording of intraoperative blood loss needs to be done jointly by surgeons and anesthesiologists, especially when the blood loss is more than $400 \mathrm{ml}$, which is conducive to perioperative fluid management.

\section{Introduction}

Perioperative fluid management is one of the key factors affecting surgical outcomes, for example, hypovolemia can lead to decreased cardiac output and tissue hypoperfusion, while hypervolemia can lead to weight gain, tissue edema, intestinal obstruction, and even death ${ }^{[1,2]}$. Intraoperative massive hemorrhage is the first independent risk factor affecting in-hospital mortality after surgery. Surgery related and patient-related factors are two possible causes of intraoperative massive hemorrhage ${ }^{[3]}$. If surgeons and anesthesiologists are able to judge whether patients are at high risk of bleeding during preoperative evaluation, then for complex cases, they can choose to perform staged surgery, use surgical techniques and anesthesia techniques during surgery and use antifibrinolytic drugs to reduce intraoperative blood loss ${ }^{[4]}$. In this study, 200 patients with lumbar spinal stenosis who underwent PLIF 
surgery were selected to study the consistency between the blood loss recorded by surgeons and anesthesiologists during the operation and the standard blood loss, aiming to provide a reference frame for proper fluid infusion and blood use in patients after surgery.

\section{Data And Methods}

General data A total of 200 cases of PLIF surgery in the Department of Spine Surgery of a Grade III Level A hospital from January 2019 to December 2020 were selected, including 109 males, aged 34-84 years, with an average of $56.40 \pm 11.21$ years, and 91 females, aged $27-78$ years, with an average of $57.48 \pm$ 11.40 years.

\subsection{Inclusion and exclusion criteria}

Inclusion criteria: (1) Patients with lumbar spinal stenosis, lumbar spondylolisthesis, or lumbar disc herniation who underwent PLIF surgery; (2) Blood routine and coagulation function were examined day before surgery; (3) Blood routine was examined on the first day after surgery.

Exclusion criteria: (1) Patients with congenital hematologic diseases or coagulation dysfunction; (2) Patients who are recently on an oral medications that affect coagulation; (3) Female patients in menstruation; (4) Patients with anemia or anemia symptoms before surgery.

\subsection{Observation indexes and evaluation of blood loss}

Observation indexes: the test results of blood routine and coagulation function before the operation, blood routine on the first day after the operation. Activated partial thromboplastin time (APTT), prothrombin time (Pt), international normalized ratio (INR), hemoglobin (HB), and hematocrit (HCT) were recorded, in addition to the blood loss and blood transfusion recorded by the operating surgeon and anesthesiologist during operation, and drainage volume on the first postoperative day.

Evaluation of blood loss: preoperative blood volume of patients was calculated according to the method proposed by Nadler ${ }^{[5]}$ in 1962: male preoperative blood volume $=0.3669 \times[$ height $(\mathrm{m})] 3+0.03219 \times$ body mass $(\mathrm{kg})+0.6041$; female preoperative blood volume $=0.3561 \times[$ height $(\mathrm{m})] 3+0.03308 \times$ body mass $(\mathrm{kg})+0.1833$. Total blood loss was calculated by gross equation: estimated total blood loss $=$ preoperative patient blood volume $\times$ (preoperative HCT - postoperative HCT) / HCT average; HCT average $=$ (preoperative HCT + postoperative HCT) / 2. Intraoperative standard blood loss = total blood loss + intraoperative blood transfusion - drainage volume on the first postoperative day.

\subsection{Statistical analysis}

Medcalc18 statistical software was used for statistical analysis. The measurement data was expressed by $(\bar{x} \pm s)$. Bland Altman method was used for comparison between the two groups with $a=0.05$ being the significance level and $\mathrm{P}>0.05$ indicating consistency between the two groups. 


\section{Results}

The patient's preoperative APTT averaged $27.27 \pm 4.90$ seconds, Pt averaged $11.83 \pm 6.58$ seconds, INR averaged $0.96 \pm 0.15, \mathrm{HCT}$ averaged $42.76 \% \pm 4.60 \%$, preoperative HB averaged $153.04 \pm 13.91 \mathrm{~g} / \mathrm{L}$ for men and $134.29 \pm 14.64 \mathrm{~g} / \mathrm{L}$ for women, all within normal range.

The difference between mean difference, standard deviation, and 95\% consistency limit between the standard blood loss and the blood loss recorded by the surgeons and the anesthesiologists were calculated separately (Table 1).

The intraoperative blood loss recorded by surgeons was less than the standard blood loss, and there was statistical difference between the intraoperative blood loss recorded by surgeons and the standard blood loss $(P<0.05)$, with no consistency (Fig. 1),the intraoperative blood loss recorded by anesthesiologists was less than the standard blood loss, and there was statistical difference between the intraoperative blood loss recorded by surgeons and the standard blood loss $(P<0.05)$, with no consistency (Fig. 2$)$.

After grouping according to the standard blood loss: when the standard blood loss was less than or equal to $400 \mathrm{ml}$, there was no statistical difference between the intraoperative blood loss recorded by surgeons and the standard blood loss ( $P>0.05$ ), which was consistent (Figure.3); When the standard blood loss was between $400 \mathrm{ml}-800 \mathrm{ml}$, the intraoperative blood loss recorded by surgeons and anesthesiologists was less than the standard blood loss,there was statistical difference between the intraoperative blood loss and the standard blood loss $(P<0.05)$, but the intraoperative blood loss recorded by surgeons was more accurate than that recorded by anesthesiologists; When the standard blood loss was more than $800 \mathrm{ml}$, the intraoperative blood loss recorded by surgeons and anesthesiologists was less than the standard blood loss, There was no consistency between the intraoperative blood loss recorded by anesthesiologists and the standard blood loss $(P<0.05)$, but the intraoperative blood loss recorded by anesthesiologists was more accurate than that recorded by surgeons. 
Table 1

corresponding mean, standard deviation and 95\% consistency limits of S-D and S-A

\begin{tabular}{|c|c|c|c|c|c|}
\hline Group & difference & $\begin{array}{l}\text { mean } \\
\text { difference }\end{array}$ & $\begin{array}{l}\text { standard } \\
\text { deviation }\end{array}$ & $\begin{array}{l}95 \% \text { upper limit } \\
\text { of consistency }\end{array}$ & $\begin{array}{l}95 \% \text { lower limi } \\
\text { of consistency }\end{array}$ \\
\hline \multirow[t]{2}{*}{ overall } & S-D & 57.48 & 63.50 & 181.93 & -66.98 \\
\hline & S-A & 69.06 & 63.04 & 192.61 & -54.50 \\
\hline \multirow{2}{*}{$\begin{array}{l}\text { Standard blood loss } \\
\leq 400 \mathrm{ml}\end{array}$} & S-D & -14.10 & 61.25 & 105.96 & -134.15 \\
\hline & S-A & 20.26 & 67.66 & 152.88 & -112.35 \\
\hline \multirow{2}{*}{$\begin{array}{l}400 \mathrm{ml}<\text { standard } \\
\text { blood loss } \leq 800 \mathrm{~m}\end{array}$} & $S-D$ & 67.88 & 4.66 & 147.58 & -11.81 \\
\hline & S-A & 84.73 & 50.19 & 183.09 & -13.64 \\
\hline \multirow{2}{*}{$\begin{array}{l}\text { Standard blood loss > } \\
800 \mathrm{ml}\end{array}$} & S-D & 99.69 & 37.48 & 173.16 & 26.23 \\
\hline & S-A & 87.05 & 54.36 & 170.65 & -19.50 \\
\hline
\end{tabular}

\section{Discussion}

Intraoperative bleeding is the most common problem in surgical practice, especially in spinal surgery, which is often accompanied by significant blood loss. A transient enhancement of fibrinolysis during surgery can lead to increased blood loss during the perioperative period of spinal surgery ${ }^{[6]}$. Relevant statistics show that the perioperative blood transfusion rate of spinal surgery ranges from $50-81 \%[4]$. There is progressive awareness of the impact of blood loss on patient outcomes. Increased blood loss means more blood transfusions are needed, exposure to more blood products increases their potential disease transmission and transfusion reaction, and massive blood loss also leads to body fluid redistribution affecting the physiological function of the heart, lung, and kidney ${ }^{[7]}$. It has been reported that blood products may damage the immune system and increase the postoperative infection rate ${ }^{[8]}$. Extensive dissection of paravertebral muscles, pedicle screw placement, laminectomy and decompression, long surgery time, and other factors all contribute to massive intraoperative blood loss, even in routine spinal surgery. An accurate assessment of intraoperative blood loss is beneficial for the guidance of fluid replacement and blood transfusion, ensuring the stability of vital signs and success of the operation. Maike Katja Kahr study have shown that in caesarean sections, the assessment of blood loss during surgery is often significantly different from the standard blood loss ${ }^{[9]}$. Overestimating blood loss during surgery can lead to excessive intravenous rehydration and blood transfusion, excessive waste of medical resources, and even blood transfusion-related complications; underestimation of blood loss during surgery can lead to miscalculation of the condition and delayed treatment, and even lifethreatening due to low blood volume shock, anemia and other related complications in patients after surgery $[10,11]$. 
Accurate evaluation of perioperative blood loss is an important basis for doctors to identify hemorrhage in patients and guide blood transfusion and fluid replacement reasonably ${ }^{[10]}$. Intraoperative blood loss is collected in the suction tank and gauze, which is often mixed with saline, flushing fluid, and other substances, causing difficulties in accurately evaluating the amount of blood loss. Intraoperative blood loss is often evaluated by weighing method, visual evaluation method, colorimetric method, hemoglobin content measurement method, shock index method, and formula method, but there is no gold standard for measuring intraoperative blood loss ${ }^{[10]}$. Sandra E Kollberg ${ }^{[12]}$ Study have shown that visual methods are inaccurate, that the amount of bleeding is often underestimated clinically, that there is a large difference between clinical evaluation and actual measurements, and that the greater the amount of bleeding, the greater the deviation in clinical estimation. Prasertcharoensuk and others compared the amount of blood loss during vaginal delivery with a visual estimate with a direct measurement, in which the rate of underrated blood loss was $89 \%[13]$, while Razvi and others found that in $57 \%$ of vaginal deliveries, visual assessment of blood loss was $20 \%$ higher than direct measurement of blood loss ${ }^{[14]}$, indicating that during surgery, clinicians typically use visual assessment. The surgical doctor can estimate the amount of bleeding according to personal experience and combined with clinical performance, but it is influenced by the evaluator's clinical experience and subjective factors, and the evaluation results are quite different ${ }^{[10,15,16]}$. This study found that when the standard blood loss was less than $400 \mathrm{ml}$, the intraoperative blood loss recorded by surgeons was consistent with the standard blood loss, but the consistency was poor; when the standard blood loss was more than $400 \mathrm{ml}$, the intraoperative blood loss recorded by surgeons was not consistent with the standard blood loss, and the intraoperative blood loss recorded by surgeons was lower than the standard blood loss. The results of this study were consistent with those of Sandra E Kollberg and other research results .

Anesthesiologists often use vital signs, $\mathrm{Hb}, \mathrm{HCT}$, complications, venous oxygen saturation, arterial oxygen saturation, and other indicators as reference basis to evaluate the blood loss of patients ${ }^{[17]}$. Toledo and others found that the error rate of anesthesiologists in estimating blood loss ranged from $16 \%$ for $300 \mathrm{ml}$ to $41 \%$ for $2000 \mathrm{ml}^{[18,19]}$. Bose and others found in their evaluation of blood loss in vaginal delivery, anesthesiologists' evaluation of blood loss was $4 \%$ higher than that of direct measurement, which was more accurate than that of helpers ${ }^{[15]}$. Our study found that there was no consistency between the intraoperative blood loss recorded by anesthesiologists and the standard blood loss. The intraoperative blood loss recorded by anesthesiologists was lower than the standard blood loss. When the standard blood loss was less than $400 \mathrm{ml}$, the intraoperative blood loss recorded by surgeons was more accurate than that recorded by anesthesiologists.

In summary, surgeons and anesthesiologists should evaluate intraoperative blood loss through a variety of methods, and gradually improve the ability to progressively evaluate intraoperative blood loss. However, this study has lack of sample size, and further increased sample size and multicenter study are needed.

\section{List Of Abbreviations}


posterior lumbar interbody fusion (PLIF); Activated partial thromboplastin time (APTT); prothrombin time (Pt); international normalized ratio (INR); hemoglobin (HB); hematocrit (HCT).

\section{Declarations}

\section{Ethics approval and consent to participate}

Not applicable.

\section{Consent for publication}

Not applicable.

\section{Availability of data and materials}

The datasets used and/or analyzed during the current study are available from the corresponding author on reasonable request.

\section{Competing interests}

All authors declare that they have no any conflict of interest.

\section{Funding}

This project is financially supported by the National Natural Science Foundation of China (NSFC81860395) $\triangle$ This project is financially supported by the National Natural Science Foundation of Ningxia (2020AAC03391); Autonomous Region Health and Health System Research Project(2019-NW011); The First-Class Discipline Construction Founded Project of NingXia Medical University and the School of Clinical Medicine (NXYLXK2017A05),

\section{Acknowledgement}

Not applicable.

\section{Author contribution}

$J \mathrm{~L}, \mathrm{HB} M$ and $\mathrm{CR} W$ carried out the studies, participated in collecting data, and drafted the manuscript. FX W and LN W performed the statistical analysis and participated in its design. XW W and NK N participated in acquisition, analysis, or interpretation of data and draft the manuscript. All authors read and approved the final manuscript.

\section{References}

1. Miller T E, Mythen M, Shaw A D, et al. Associations between perioperative fluid management and patient outcomes: a multicentre retrospective study.[J]. British journal of anaesthesia,2020. 
2. Miller T E, Myles P S. Perioperative Fluid Therapy for Major Surgery[J]. Anesthesiology,2019,130(5):825-832.

3. Kushioka J, Takenaka S, Makino T, et al. Risk factors for in-hospital mortality after spine surgery: a matched case-control study using a multicenter database[J]. Spine J, 2020,20(3):321-328.

4. Bible J E, Mirza M, Knaub M A. Blood-loss Management in Spine Surgery[J]. J Am Acad Orthop Surg,2018,26(2):35-44.

5. Piscitelli P, lolascon G, Innocenti M, et al. Painful prosthesis: approaching the patient with persistent pain following total hip and knee arthroplasty[J]. Clin Cases Miner Bone Metab,2013,10(2):97-110.

6. Yoo J S, Ahn J, Karmarkar S S, et al. The use of tranexamic acid in spine surgery[J]. Ann Transl Med,2019,7(Suppl 5):S172.

7. Hu S S. Blood loss in adult spinal surgery[J]. Eur Spine J,2004,13 Suppl 1:S3-S5.

8. Chow J H, Chancer Z, Mazzeffi M A, et al. Impact of Preoperative Platelet Count on Bleeding Risk and Allogeneic Transfusion in Multilevel Spine Surgery[J]. Spine (Phila Pa 1976),2021,46(1):E65-E72.

9. Kahr M K, Brun R, Zimmermann R, et al. Validation of a quantitative system for real-time measurement of postpartum blood loss[J]. Arch Gynecol Obstet,2018,298(6):1071-1077.

10. Guinn N R, Broomer B W, White W, et al. Comparison of visually estimated blood loss with direct hemoglobin measurement in multilevel spine surgery[J]. Transfusion,2013,53(11):2790-2794.

11. Maio M, Carvalho A, Pinho A, et al. What Factors can Influence Massive Blood Loss in the Surgical Treatment of Neuromuscular Scoliosis?[J]. Rev Bras Ortop (Sao Paulo),2020,55(2):181-184.

12. Kollberg S E, Haggstrom A E, Lingehall H C, et al. Accuracy of Visually Estimated Blood Loss in Surgical Sponges by Members of the Surgical Team[J]. AANA J,2019,87(4):277-284.

13. Schorn M N. Measurement of blood loss: review of the literature[J]. J Midwifery Womens Health,2010,55(1):20-27.

14. Razvi K, Chua S, Arulkumaran S, et al. A comparison between visual estimation and laboratory determination of blood loss during the third stage of labour[J]. Aust N Z J Obstet Gynaecol,1996,36(2):152-154.

15. Bose P, Regan F, Paterson-Brown S. Improving the accuracy of estimated blood loss at obstetric haemorrhage using clinical reconstructions[J]. BJOG,2006,113(8):919-924.

16. Yoong W, Karavolos S, Damodaram M, et al. Observer accuracy and reproducibility of visual estimation of blood loss in obstetrics: how accurate and consistent are health-care professionals?[J]. Arch Gynecol Obstet,2010,281(2):207-213.

17. Joyce J A. Toward reducing perioperative transfusions.[J]. AANA journal,2008,76(2).

18. Adkins A R, Lee D, Woody D J, et al. Accuracy of blood loss estimations among anesthesia providers[J]. AANA J,2014,82(4):300-306.

19. Toledo P, Mccarthy R J, Hewlett B J, et al. The accuracy of blood loss estimation after simulated vaginal delivery[J]. Anesth Analg,2007,105(6):1736-1740. 
Figures

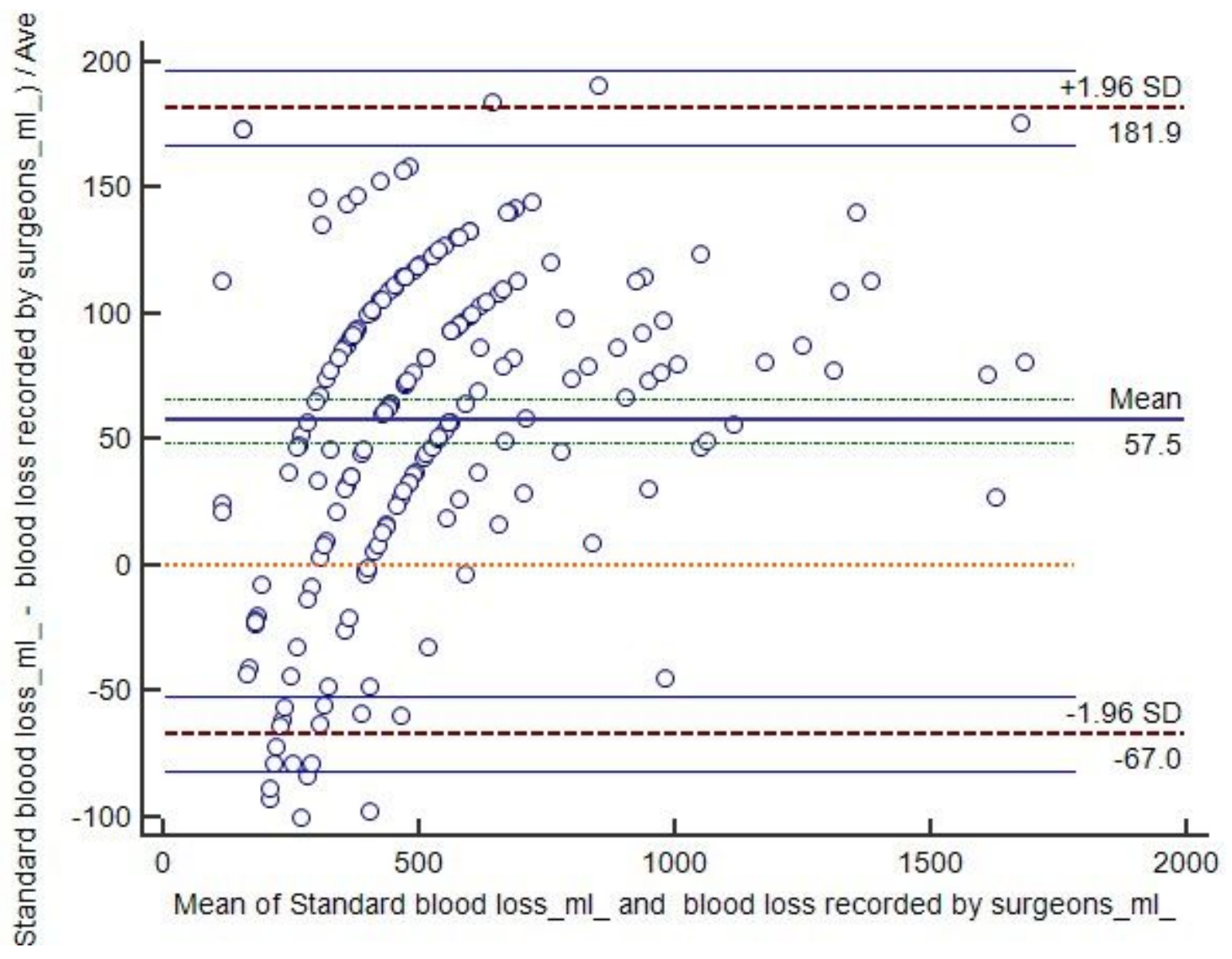

\section{Figure 1}

The intraoperative blood loss recorded by surgeons was less than the standard blood loss, and there was statistical difference between the intraoperative blood loss recorded by surgeons and the standard blood loss $(P<0.05)$, with no consistency 


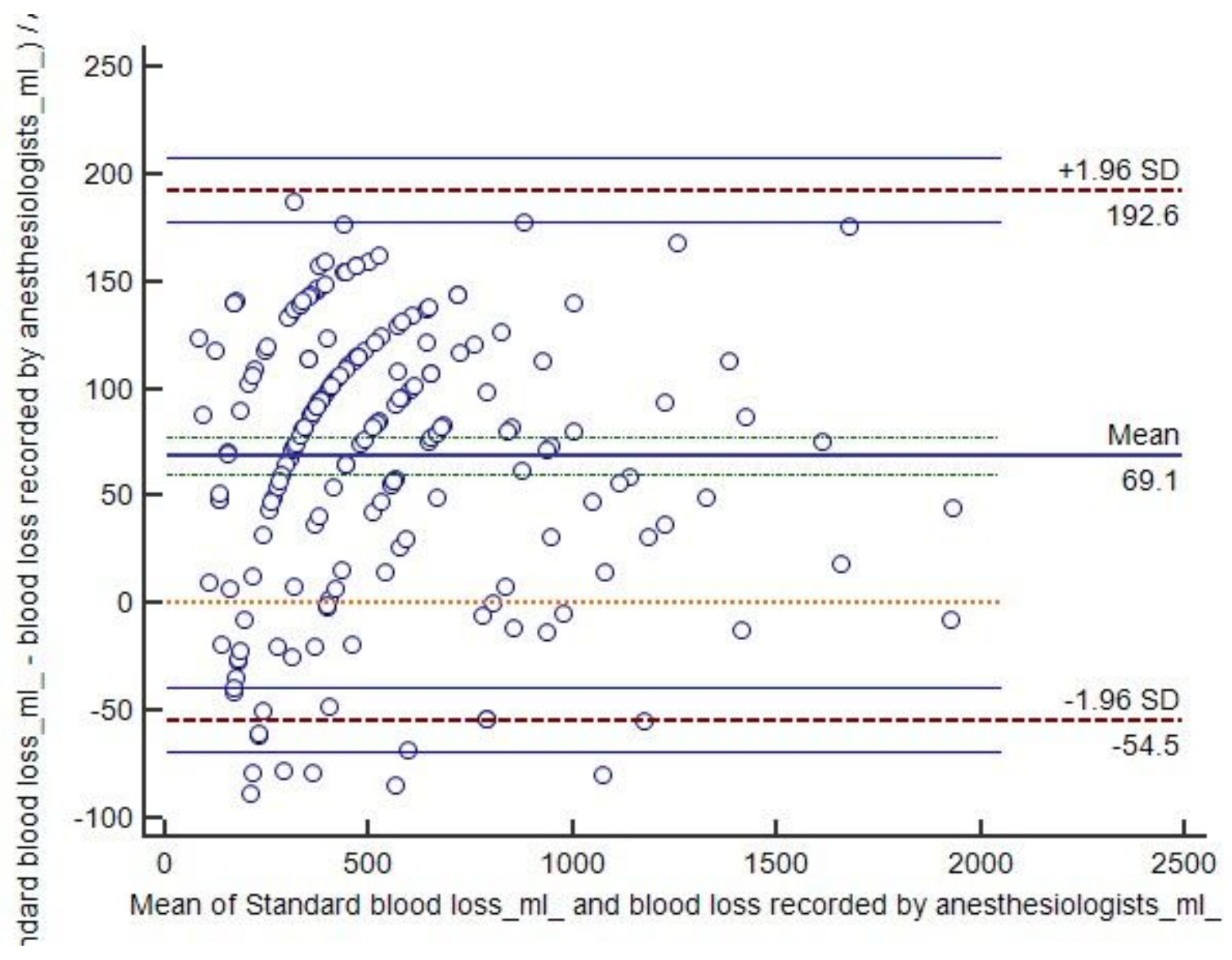

\section{Figure 2}

The intraoperative blood loss recorded by anesthesiologists was less than the standard blood loss, and there was statistical difference between the intraoperative blood loss recorded by surgeons and the standard blood loss $(P<0.05)$, with no consistency 


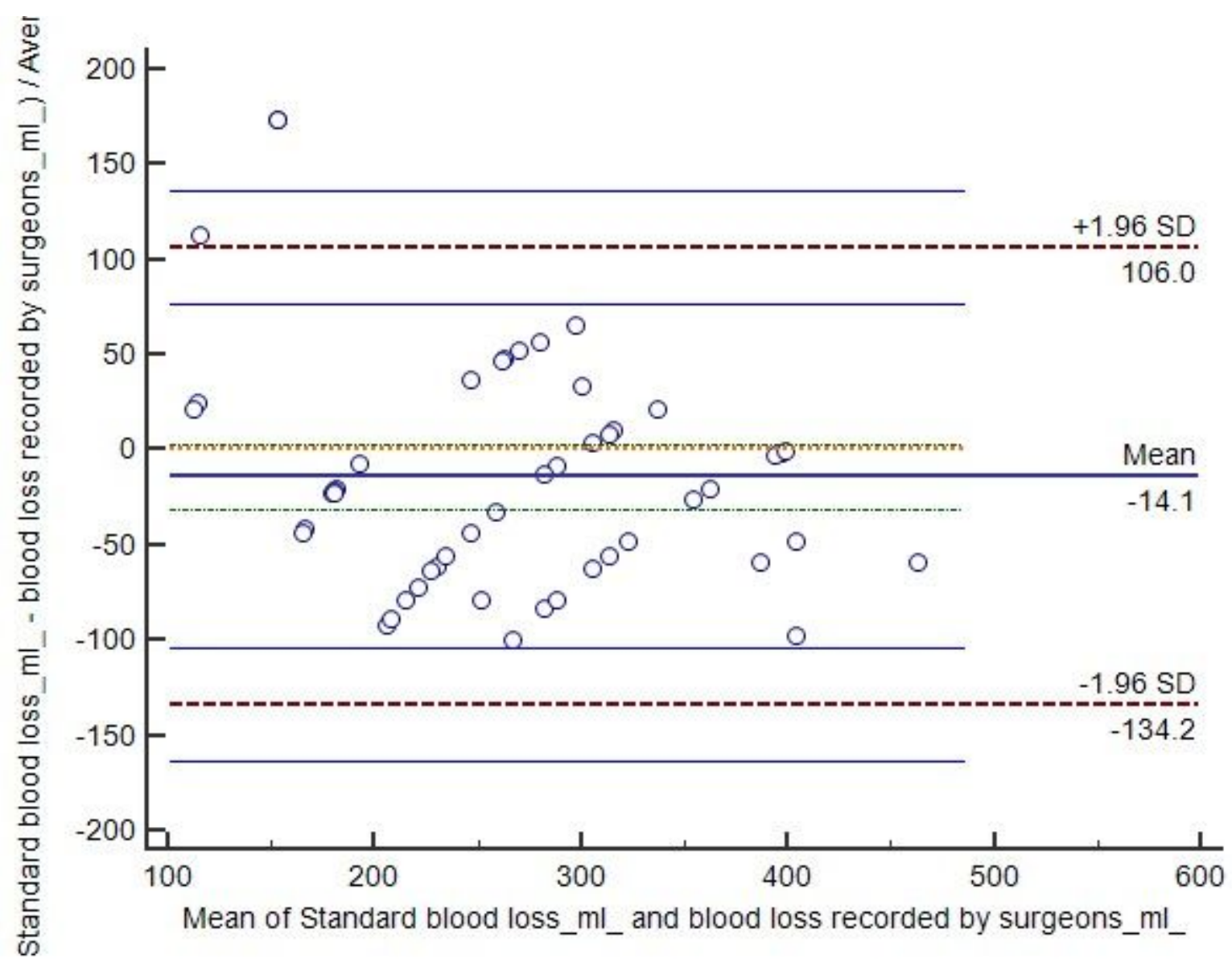

\section{Figure 3}

After grouping according to the standard blood loss: when the standard blood loss was less than or equal to $400 \mathrm{ml}$, there was no statistical difference between the intraoperative blood loss recorded by surgeons and the standard blood loss $(P>0.05)$, which was consistent 